\title{
Use of a Mid-Infrared Free-Electron Laser (MIR-FEL) for Dissociation of the Amyloid Fibril Aggregates of a Peptide
}

\author{
Takayasu Kawasaki, Takayuki Imai, Koichi Tsukiyama \\ IR Free Electron Laser Research Center, Research Institute for Science and Technology (RIST), Tokyo University \\ of Science, Chiba, Japan \\ Email: kawasaki@rs.tus.ac.jp
}

Received 21 January 2014; revised 21 February 2014; accepted 28 February 2014

Copyright (C) 2014 by authors and Scientific Research Publishing Inc.

This work is licensed under the Creative Commons Attribution International License (CC BY).

http://creativecommons.org/licenses/by/4.0/

(c) (i) Open Access

\section{Abstract}

Amyloid fibrils are deposited in various tissues in the body, and are linked to the putative causes of serious diseases such as amyloidosis. Although the conditions of the disease would be expected to improve if the fibril structure could be destroyed, the aggregated structure is stable under physiological conditions. Recently, we found that the amyloid fibrils of lysozyme could be refolded into their active form by using a mid-infrared free-electron laser (MIR-FEL) tuned to the amide I band (corresponding to the $\mathrm{C}=0$ stretch vibration), with the MIR-FEL having specific oscillation characteristics of a picosecond pulse structure, a tunable wavelength within mid-infrared frequencies, and high photon density. In the study, we tested the usability of the FEL for dissociation of aggregates of pathological amyloid fibrils by using a short peptide of human thyroid hormone. The fibrils (after being placed on a glass slide) were irradiated using the FEL tuned to the amide I band $\left(1644 \mathrm{~cm}^{-1}\right)$, and those in situ were analyzed by Congo-Red assay, scanning-electron microscopy, and transmission-electron microscopy. All of the results obtained using these microscopic analyses indicated that the amyloid fibril formation was considerably decreased by FEL irradiation. Moreover, upon irradiation, a strong fibril peak at the amide I band in the infrared spectrum was transformed into a broad peak. These results imply that the $\beta$-sheet-rich structure of the amyloid fibrils changed into non-ordered or unspecified structures after the FEL irradiation. This FEL irradiation system, combined with various analytical methods, shows promise for the dissociation of amyloid aggregates.

\section{Keywords}

Amyloid Fibrils; Amyloidosis; Free-Electron Laser; Amide I Band; $\beta$-Sheet 


\section{Introduction}

Amyloid fibrils are widely recognized to be formed by diverse polypeptides, such as amyloid $\beta$, tau protein, polyglutamine, prion protein, insulin, $\alpha$-synuclein, and $\beta_{2}$-microglobulin, and they are deposited in the tissues of various organs during amyloidosis [1]-[8]. Those fibrils are commonly composed of $\beta$-sheet-rich structures and insoluble in the physiological solution. However, the detailed mechanism by which amyloid fibrils form, and effective methods for the treatment of amyloidosis in diseases such as myeloma are not yet fully established [9]. Although the disease conditions may be ameliorated by reducing the amount of amyloid fibrils found in pathological tissues, the fibrils are robust structures under physiological conditions, and are disaggregated only when denaturants such as guanidine hydrochloride and organic solvents such as dimethyl sulfoxide (DMSO) are used [10]. These reagents are toxic for the human body and are not suitable for the treatment of the disease.

We have developed a mid-infrared free-electron laser (MIR-FEL) at the Tokyo University of Science (FELTUS) as shown in Figure 1. The oscillation wavelengths of the FEL are tunable within the 5.0 - $16 \mu \mathrm{m}$ range, which corresponds to the absorption frequencies of the vibrational modes of various molecules [11]-[14]. In addition, the FEL features a high-powered, picosecond pulse structure of oscillation, and therefore has been used previously to analyze biological molecules [15]-[17]. Recently, we found that the amyloid fibrils of lysozyme could be refolded into their active state using FEL irradiation tuned to the amide I band [18]. Although the fibril structures were dissociated, the primary structure was not destroyed, as indicated by microscopic analyses and biochemical conventional methods such as electrophoresis. The amide $\mathrm{C}=\mathrm{O}$ stretching vibration is known to be affected by fibrillation, a process during which hydrogen-bond networks form between $\beta$-sheet structures in the fibril state [19]. Thus, it can be considered that the FEL oscillation tuned to the amide I band can dissociate this network of non-covalent bonds, and drive the refolding of the fibril into the native state. For confirming the effects of FEL on amyloid fibrils, and to establish a general method for dissociation of pathological aggregates using the FEL, additional supporting data are required.

In this study, we targeted a small peptide, Asp-Phe-Asn-Lys-Phe (DFNKF), which can form amyloid fibrils under physiological conditions. This peptide exists as an internal sequence of calcitonin hormone, and the amyloid fibrils of the peptide cause medullary carcinoma of the thyroid [20]. This short peptide sequence is considered to have adequate correlation between hydrophobicity and hydrophilic elements for fibril formation. By using this peptide, we have validated the effect of the FEL on the structural change of the amyloid fibrils, and examined the use of the FEL irradiation system for dissociation of amyloid aggregates.

\section{Materials and Methods}

\subsection{Materials}

All of the reagents used in this study were purchased as special-grade chemicals from various companies. Acetonitrile was purchased from Nacalai Tesque, Inc. (Kyoto, Japan). Congo Red (CR) and trans-2-[3-(4-tert-butylphenyl)-2-methyl-2-propenylidene] malononitrile (DCTB) were purchased from Sigma-Aldrich (Tokyo, Japan). Tris-base, DMSO, hydrochloric acid ( $\mathrm{HCl}$ ), phosphate buffered saline (PBS, $10 \mathrm{mM}$ ), and sodium chloride ( $\mathrm{NaCl}$ ) were purchased from Wako Pure Chemical Industries (Osaka, Japan). The KBr mini-plate was purchased from Jasco Engineering Co. (Tokyo, Japan). The synthesized pentapeptide, DFNKF, (80.9\% purity) was purchased from PH Japan Co., Ltd. (Hiroshima, Japan).

\subsection{Mid-Infrared Free-Electron Laser Facility at the Tokyo University of Science (FEL-TUS)}

The FEL-TUS can generate a laser beam using a synchrotron radiation (SR) as a seed, with a variable wavelength in the mid-infrared region (5.0 - $16 \mu \mathrm{m}, 625-2000 \mathrm{~cm}^{-1}$ ) (Figure 1(a)). An electron beam generated by a high radio frequency (RF) electron gun $(2856 \mathrm{MHz})$ is accelerated by a linear accelerator and injected into an undulator (a periodic magnetic field). The electron beam is forced to oscillate in the undulator to generate SR. The maximum acceleration energy is $40 \mathrm{MeV}$. Light of a specific wavelength satisfying the following equation is amplified by interaction between the generated SR and the electron beam:

$$
\lambda=\frac{\lambda \mu}{2 \gamma^{2}}\left[1+\frac{K^{2}}{2}\right]
$$


(a)

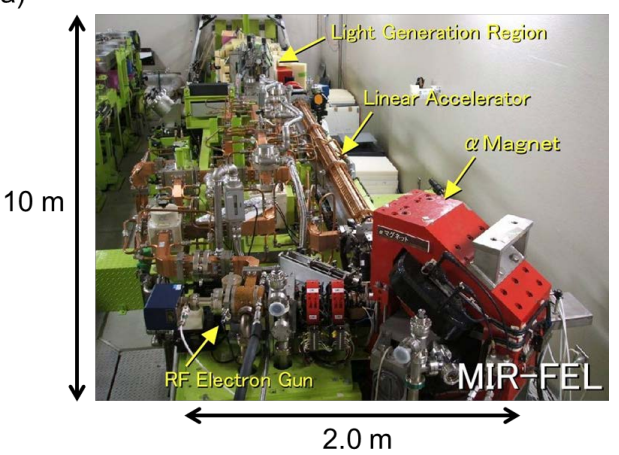

(b)

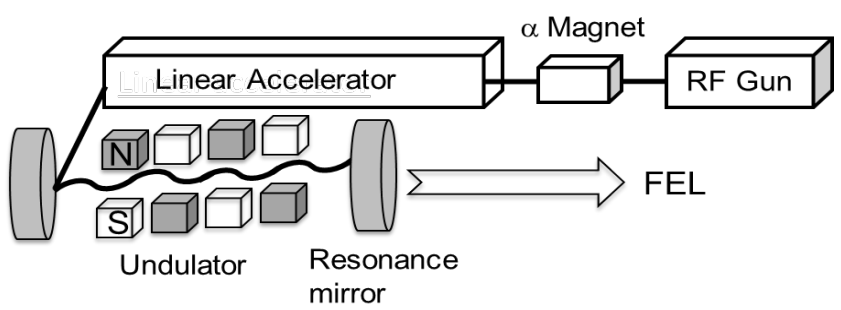

Figure 1. Mid-infrared free-electron laser (MIR-FEL) generation system. (a) The FEL control room: width, 2.0 m; depth, 10 $\mathrm{m}$. The room is a compact-sized photon factory; (b) A schematic drawing of the FEL oscillation. The laser beam is generated using an electronic gun and is accelerated to $40 \mathrm{MeV}$ through the linear accelerator. The periodic length of the undulator is tunable, and the FEL is transported to the laboratory though the vacuum tube and controlled by the mirror.

where " $\lambda$ " is the FEL wavelength to be amplified, " $\lambda \mathrm{u}$ " is the periodic length of the undulator, " $\gamma$ " is proportional to the acceleration energy of the electron beam, and " $\mathrm{K}$ " is proportional to the strength of the periodic magnetic field. In this study, the wavelength of the oscillation beam was tuned to $6.08 \mu \mathrm{m}\left(\mathrm{ca} .1644 \mathrm{~cm}^{-1}\right)$ by adjusting the space interval of the undulator to $13.6 \mathrm{~mm}$, and that was tuned to $5.0 \mu \mathrm{m}\left(2000 \mathrm{~cm}^{-1}\right)$ by adjusting the space interval of undulator to $15.8 \mathrm{~mm}$ for comparison.

The maximum value of periodic magnetic flux density is set to $0.83 \mathrm{~T}$, and the amplified SR is reflected upstream of the electron beam by a mirror positioned downstream of the beam, and then re-reflected by the upstream mirror to interact with the electron beam again, producing a coherent laser light. FEL-TUS provides two types of laser pulses: a macro-pulse and micro-pulse. The macro-pulse has a duration of $\sim 2 \mu \mathrm{s}$, and has a repetition rate of $5 \mathrm{~Hz}$ throughout the operation; it consists of a train of micro-pulses with durations of 2 ps each, and an interval of 350 ps between two consecutive micro-pulses. The energy of the laser pulse used for the current experiment was in the range of 6.0 - $8.0 \mathrm{~mJ}$ per macro-pulse, which was measured using an energy meter (SOLO2, Gentec-EO Inc., Quebec, Canada).

\subsection{Preparation of Amyloid Fibrils and Irradiation with IR-FEL}

The synthesized peptide was dissolved in 10\% DMSO in buffer A, which contained 10 mM of tris-base and 20 $\mathrm{mM}$ of $\mathrm{NaCl}$; the $\mathrm{pH}$ of the solution was adjusted to 7.5 using $\mathrm{HCl}$. The peptide powder was dissolved at a concentration of $100 \mathrm{mg} / \mathrm{mL}$ in DMSO, then diluted to $10 \mathrm{mg} / \mathrm{mL}$ using buffer $\mathrm{A}$, and the solution was incubated for two days at $37^{\circ} \mathrm{C}$. The resulting solution $(100 \mu \mathrm{L})$ containing amyloid fibrils was placed on a glass slide (Matsunami Glass Ind., Ltd, Osaka, Japan) and irradiated with the IR-FEL (tuned to various wavelengths) at $37^{\circ} \mathrm{C}$. To prevent the water from evaporating, $10 \mu \mathrm{L}$ of water was added periodically to the suspension during the irradiation process. The temperature on the glass slide was measured by using a thermometer during the irradiation. After irradiation was complete, the sample, while still on the glass slide, was air-dried and subjected to various analyses.

\subsection{Congo-Red (CR) Assay}

CR produces green-yellow birefringence in the presence of fibrils [21]. CR solution (10 $\mu \mathrm{L}, 0.2 \mathrm{mM}$ in PBS) was added to the amyloid fibrils on glass slides, which were then incubated for 10 min at room temperature. After the sample was air-dried, the birefringence was observed using polarized light microscopy (MVX 10, Olympus, Tokyo, Japan).

\subsection{Scanning Electron Microscopy (SEM)}

The morphologies of the amyloid fibrils of DFNKF were analyzed using an FE-SEM Supra 40 scanning electron microscope (Carl Zeiss). After generating amyloid fibrils as described above, $100 \mu \mathrm{L}$ of the fibril solution was 
placed on a glass slide and air-dried. The glass slide was fixed on a sample holder by using conductive copper tape, and the acceleration voltage was set to $7.00 \mathrm{kV}$.

\subsection{Transmission Electron Microscopy (TEM)}

Specimens for TEM observation were prepared as follows: Firstly, $2 \mu \mathrm{L}$ of each peptide sample was deposited on copper grids (Nisshin EM, 200 mesh) covered with collodion film hydrophilized by an electric glow discharge. After $30 \mathrm{~s}$ of deposition, an excess amount of the sample was blotted with a filter paper, followed by two more deposition-blotting cycles with $20 \mu \mathrm{L}$ of water, and two more cycles with $25 \mu \mathrm{L}$ of $1 \mathrm{wt} \%$ phosphotungstic acid. Prior to sample preparation, the staining solution was filtered through a $0.22-\mu \mathrm{m}$ membrane filter to remove any large crystals. The TEM observation was performed by using a Hitachi H-7650 instrument at $120 \mathrm{kV}$ of accelerating voltage.

\subsection{Fourier-Transform Infrared Spectroscopy (FT-IR)}

FT-IR spectra were recorded on an FT-IR 615 spectrophotometer (Jasco International Co., Ltd., Tokyo, Japan) using a solid $\mathrm{KBr}$ mini-plate. The peptide sample was mixed with the $\mathrm{KBr}$ pellet and a thin plate was prepared. Spectra were measured using 16 scans at a resolution of $4 \mathrm{~cm}^{-1}$. Parameters of amide I band were calculated based on the software, Spectra Manager for Windows 95/NT (Jasco International Co., Ltd., Tokyo, Japan). Normalization of spectra was performed by using Spectra Manager software Ver.2 (Jasco International Co., Ltd., Tokyo, Japan).

\subsection{Matrix-Assisted Laser Desorption-Time-Of-Flight Mass Spectrometry (MALDI-TOF MS)}

Mass spectra were acquired using a MALDI-TOF mass spectrometer (SpiralTOF JMS-S3000, JEOL). The sample on a glass slide was dissolved in acetonitrile $(\sim 50 \mu \mathrm{L})$, mixed with a solution of DCTB in the same solvent, and an aliquot $(\sim 2 \mu \mathrm{L})$ was transferred to the MALDI plate. After the samples were dried, mass spectra were measured in spiral and positive modes.

\section{Results}

The MIR-FEL generation system is shown in Figure 1(a). The instrument is composed of an RF gun, an $\alpha$ magnet, a linear accelerator, and a light generation region; this system is installed in a small-scale photon factory. The overall experimental design used in this study was similar to that employed in the previous study [12]. In brief, the laser was generated by the high-frequency electron gun, passed through the linear accelerator, and oscillated in the undulator (Figure 1(b)). The specific coherent light was amplified by the interaction between the generated SR and the electron beam, and the laser was directed by the gold-coated mirror onto the sample. The method used for irradiating the sample is shown in Figure 2(a). The sample was placed on a glass slide and di rectly irradiated by the FEL. After being irradiated for two hours, the samples were air-dried and analyzed in situ by the various microscopic instruments described below. Although the irradiation time was relatively long, the temperature increase on the glass slide was only two or three degrees. Prior to the FEL irradiation, the infrared spectrum of the amyloid fibrils of the peptide was measured to determine the oscillation wavelength of the FEL (Figure 2(b)). The center peak intensity at $c a .1643 \mathrm{~cm}^{-1}$ of P2 of the fibrils (spectrum 1) was comparatively higher than that at $c a .1641 \mathrm{~cm}^{-1}$ of P2 of the peptide before fibrillation (spectrum 2). The parameters of the amide I bands consisting of P2 and P1 at $1673 \mathrm{~cm}^{-1}$ are summarized in Table 1, in which the peak area ratio (P2/P1) of the fibrils (spectrum 1) was larger than that of the peptide before fibrillation (spectrum 2). This increase in the infrared absorption peak intensity at the lower wave number of the amide I band (P2) is caused by a formation of antiparallel $\beta$-sheet structure, which is consistent with previous experimental result [20]. To examine the effects of the tuning of FEL to the amide I band on the structural conversion of peptide fibrils, the peak of P2 was targeted for FEL irradiation, and the oscillation wavelength was tuned to $6.08 \mu \mathrm{m}$, (ca. 1644 $\left.\mathrm{cm}^{-1}\right)$ as described in the experimental section. In addition, a beam at a frequency of $2000 \mathrm{~cm}^{-1}(5.0 \mu \mathrm{m})$, where no major peaks existed, was used in a separate experiment for comparison purposes.

After the amyloid fibrils were irradiated by the FEL, they were stained with CR and observed using polarized light microscopy (Figure 3). In the dried fibril sample, birefringence was observed as a bright green-yellow col- 
(a)
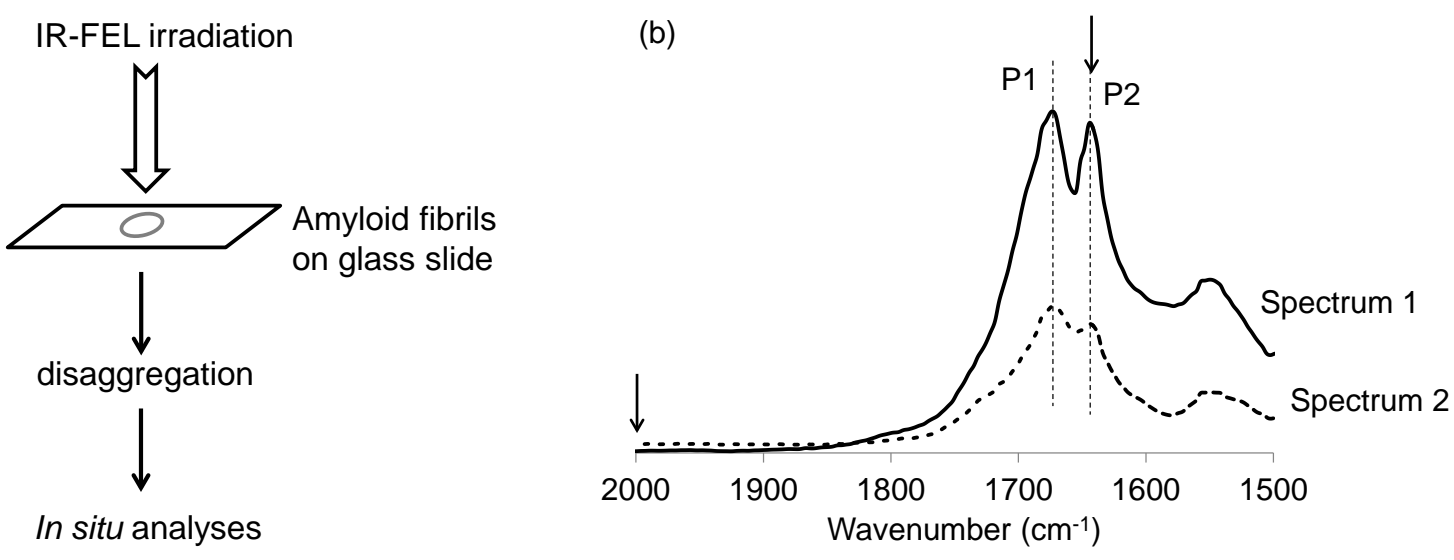

Figure 2. The irradiation method for dissociation of amyloid fibrils. (a) The amyloid fibrils were placed on a glass slide and, after FEL irradiation, analyzed in situ by various microscopic methods. (b) FT-IR spectra of amyloid fibrils of DFNKF. Spectrum 1: The infrared spectrum of the amyloid fibrils. Spectrum 2: The spectrum of the peptide before fibrillation. The frequencies targeted by the FEL are marked by arrows $\left(1644.7 \mathrm{~cm}^{-1}(6.08 \mu \mathrm{m})\right.$ and $\left.2000 \mathrm{~cm}^{-1}(5.0 \mu \mathrm{m})\right)$.

Table 1. Parameters of the amide I bands.

\begin{tabular}{|c|c|c|c|c|c|c|c|c|c|}
\hline & \multicolumn{4}{|c|}{ P1 $\left(1655.6\right.$ - $\left.1699.9 \mathrm{~cm}^{-1}\right)$} & \multicolumn{4}{|c|}{ P2 (1619.9 - $\left.1655.6 \mathrm{~cm}^{-1}\right)$} & \multirow{3}{*}{$\begin{array}{c}\text { Area ratio } \\
\text { (P2/P1) }\end{array}$} \\
\hline & & Center $\mathrm{F}$ & eak & & & Center & & & \\
\hline & $\left(\mathrm{cm}^{-1}\right)$ & height & width & area & $\left(\mathrm{cm}^{-1}\right)$ & height & width & area & \\
\hline Spectrum 1 & 1673.91 & 0.285 & 23.82 & 6.879 & 1643.05 & 0.284 & 15.80 & 4.734 & 0.688 \\
\hline Spectrum 2 & 1673.91 & 0.115 & 23.05 & 2.757 & 1641.12 & 0.081 & 13.60 & 1.256 & 0.456 \\
\hline
\end{tabular}

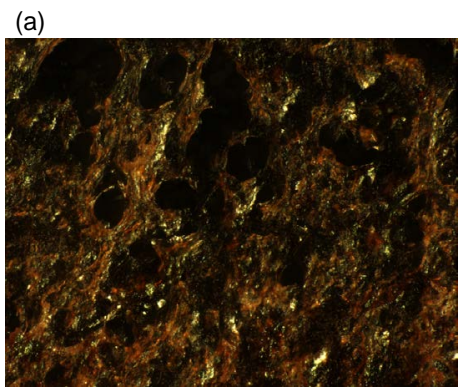

(b)

(c)

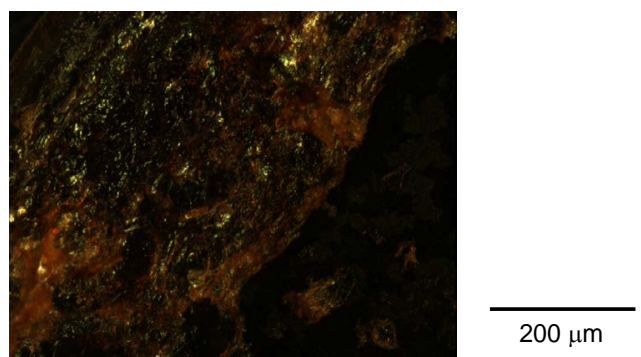

Figure 3. Congo red (CR) staining of the amyloid fibrils of DFNKF. The CR reagent was mixed with the fibrils on a glass slide, and the birefringence was observed using polarized light microscopy. (a) Amyloid fibrils; (b) Fibrils after FEL irradiation at $6.08 \mu \mathrm{m}$; and (c) Fibrils after FEL irradiation at $5.0 \mu \mathrm{m}$. 
or (Figure 3(a)), due to binding of CR into the $\beta$-sheet structures of the amyloid fibrils [21]. However, after the FEL was tuned to $6.08 \mu \mathrm{m}$, the brightness of the fibrils decreased dramatically (Figure 3(b)). In contrast, the brightness largely remained when the fibrils were irradiated by the FEL tuned to $5.0 \mu \mathrm{m}$ (Figure 3(c)). These observations indicate that FEL tuned to the amide I band reduced the amyloid fibrils of the peptide, whereas the beam at $5.0 \mu \mathrm{m}$ was ineffective. Because the energy levels of the FELs were very similar (within 6.0 - $8.0 \mathrm{~mJ}$ ), this difference indicates wavelength specificity when the FEL is used to promote the dissociation of amyloid fibrils.

The structural changes in the fibrils were observed in situ using SEM. After the FEL irradiation at $6.08 \mu \mathrm{m}$ was completed, the sample on the glass slide was air-dried and immediately transferred into the chamber of the SEM instrument. The amyloid fibrils of the peptide contained numerous silk-like filaments, whose lengths were longer than $10 \mu \mathrm{m}$ (Figure 4(a)). After FEL irradiation, the filaments disappeared almost completely, and a number of small boluses were observed (Figure 4(b)). Although peptide conformations are, in general, varied (making it difficult to identify non-fibrillar solids of the peptide), this image indicates that the amyloid fibrils were decreased after being irradiated by the FEL tuned to the amide I band.

The structural changes in fibrils after FEL irradiation was also observed by TEM at high spatial resolution. The peptide fibrils were prepared, and then irradiated by the FEL in the manner described above. As shown in Figure 4(c), several negatively stained thin strings (several nanometers in width) were observed, with lengths ranging from $50 \mathrm{~nm}$ to $200 \mathrm{~nm}$. After FEL irradiation, those strings were decreased, and fuzzy, circular forms appeared (Figure 4(d)). Although it is difficult to determine if these fuzzy forms are non-ordered conformers of the peptide, this TEM image indicates that almost amyloid fibrils of the peptide were disaggregated after being irradiated by the FEL. Next, the fibril samples were analyzed by FT-IR to examine the secondary conformation of the peptide.

The infrared amide I band region (1600 - $\left.1700 \mathrm{~cm}^{-1}\right)$ of amyloid fibrils of the peptide was measured (Figure 5(a)). The peak intensity at $6.08 \mu \mathrm{m}$ in the fibrils (black solid line) was higher than that of the broad peak of

(a)

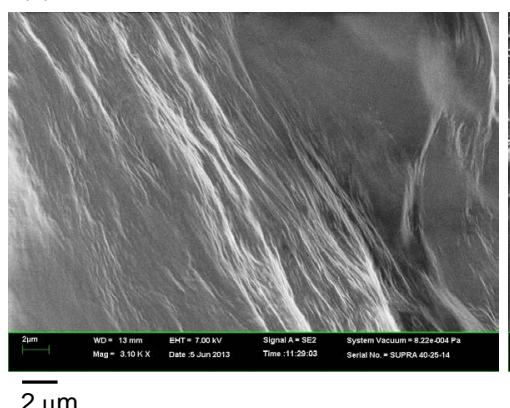

(c)

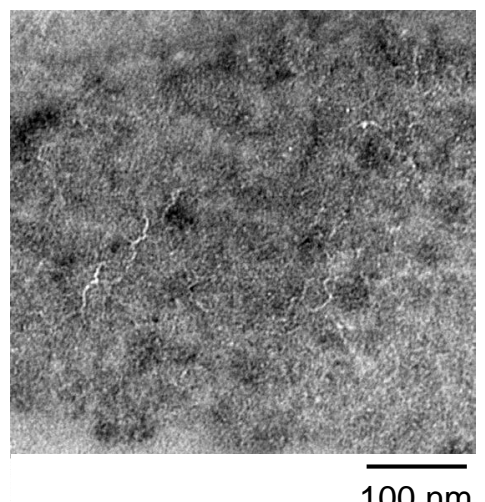

(b)

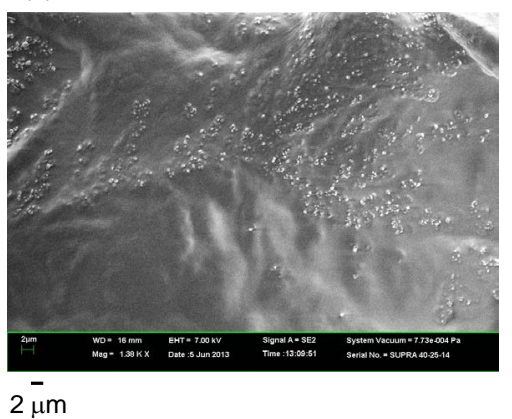

(d)

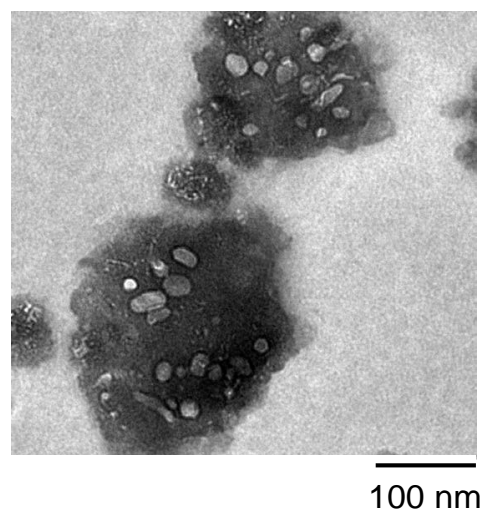

Figure 4. Electron microscopic analyses. (a) Scanning electron microscopy (SEM) image of amyloid fibrils; (b) SEM image of the fibrils after FEL irradiation; (c) Transmission electron microscopy (TEM) image of amyloid fibrils; (d) TEM image of the fibrils after FEL irradiation. 
(a)
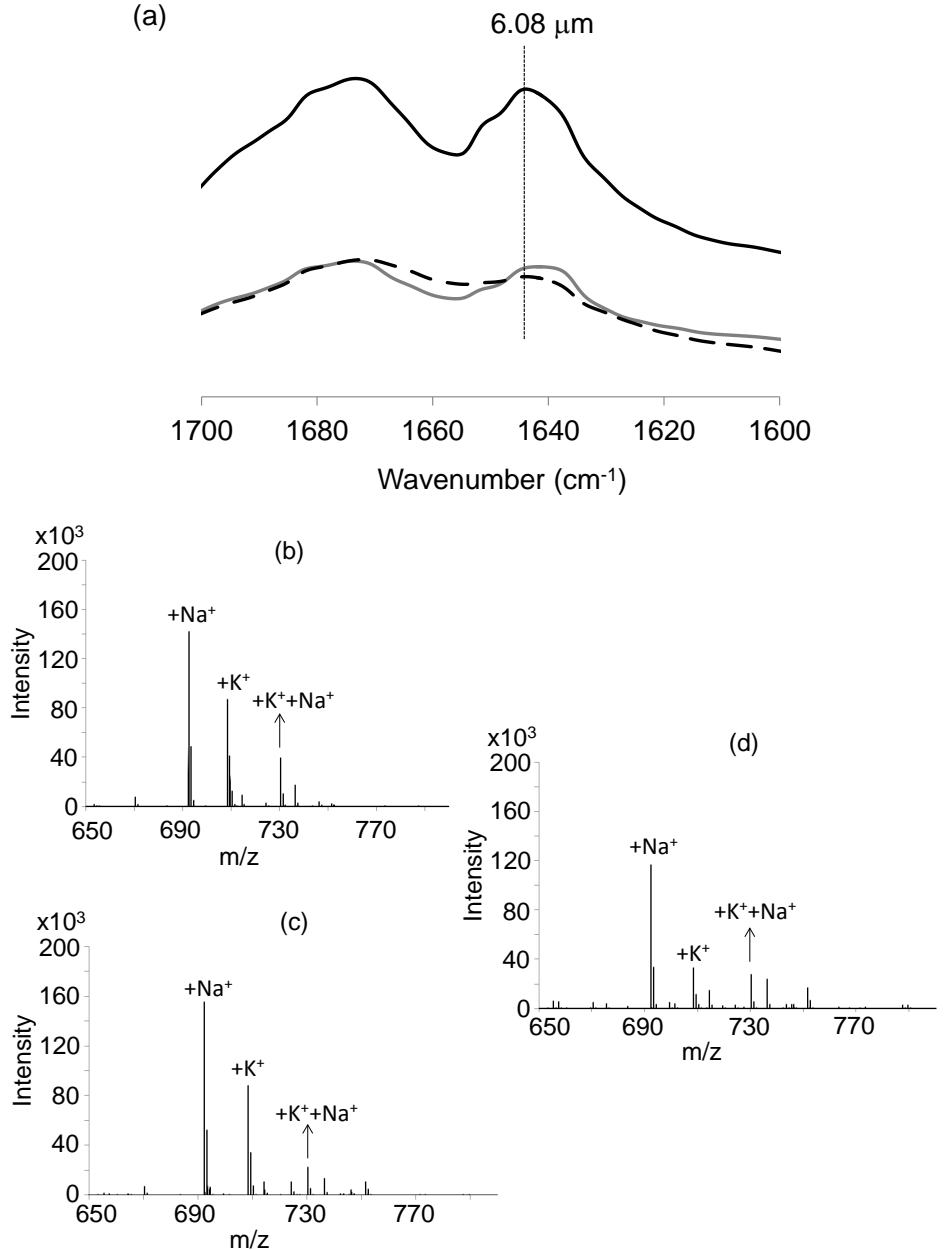

Figure 5. Structural analyses of amyloid fibrils after FEL irradiation. (a) FT-IR spectra of the amide I region: solid line, spectrum of fibrils; dashed line, spectrum of the peptide before fibrillation; gray line, spectrum of the fibril after FEL irradiation; (b)-(d) MALDI-TOF MS spectra in the positive mode. (b) Mass spectrum of the peptide (DFNKF) before fibrillation: calculated MW, $669.74 \mathrm{Da}$. (c) Mass spectrum of the amyloid fibrils formed by the peptide. (d) Mass spectrum of the amyloid fibrils following FEL irradiation. Trans-2-[3-(4-tert-butylphenyl)-2-methyl-2-propenylidene] malononitrile was used as the matrix.

peptide before fibrillation (black dashed line), which indicates that the peptide was modified into the fibril structure with anti-parallel $\beta$-sheets, as described above [22]. After the fibrils were irradiated by the FEL at 6.08 $\mu \mathrm{m}$, the strong peak was diminished to a broad peak (gray line). This broad peak indicates that strict $\beta$-sheet structures of the fibrils were changed into non-ordered or random conformations of the peptide after FEL irradiation, and the intermolecular hydrogen bonds formed by amide backbones in $\beta$-sheet structures could be easily cleaved by FEL irradiation tuned to the amide I band.

Finally, we sought to confirm that the primary structure of the peptide was not affected by FEL irradiation, by obtaining the mass spectrum of the peptide (Figures 5(b)-(d)). The peptide sample was placed on a glass slide, and mixed with the matrix in acetonitrile, and an aliquot was transferred onto the MALDI plate. The mass of the peptide before fibrillation (calculated MW: 669.74) was detected as a sodium ion adduct $(+22)$ in the positive mode (measured: 692.44; calculated: 692.74), accompanied by a potassium ion adduct $(+38)$ (measured: 708.41; calculated: 708.74), and an adduct containing a sodium ion and a potassium ion (measured: 730.39; calculated: 730.74) (Figure 5(b)). The mass spectrum of the amyloid fibrils was obtained in the same way as for the peptide 
before fibrillation (Figure 5(c)). The mass spectrum of the fibrils after FEL irradiation at $6.08 \mu \mathrm{m}$ was almost identical to the spectrum of the non-irradiated fibrils (Figure 5(d)). Although we were unable to analyze the spectra in detail below $600 \mathrm{Da}$, no major differences were detected between the mass spectrum patterns of fibrils with and without FEL irradiation. These results indicate that the FEL irradiation affected the higher-order structure of the peptide without destroying the primary structure.

\section{Discussion}

In a previous study, we found that IR-FEL tuned to the amide I band could promote the refolding of amyloid fibrils of lysozyme into their active form [18]. As for the specific refolding mechanism, we proposed that FEL irradiation of the amyloid fibrils heated the amide bonds and surrounding water molecules, to drive the refolding of fibrils into their native form [23] [24]. Amyloid fibrils can, in general, be refolded in the presence of denaturants such as guanidine hydrochloride, and the refolding can be initiated from non-ordered or random structures after removal of the denaturant [10] [25]. However, the mechanism underlying the refolding induced by the FEL method is likely distinct from that of refolding triggered by those conventional methods. Herein, we tested this hypothetical mechanism by using a peptide fragment ( $c a .700 \mathrm{Da}$ ), which can form amyloid fibrils similar to the lysozyme protein $(14 \mathrm{kDa})$, although their molecular sizes are different. The FEL irradiation that targeted the $\mathrm{C}=\mathrm{O}$ stretching vibration mode of the $\beta$-sheet-rich structure of the peptide fibrils was able to dissociate the fibril structure into a non-ordered state without destroying the primary structures, although this effect was dependent on the oscillation wavelength. These results support the mechanism of the dissociation of amyloid fibrils induced by the FEL proposed in our previous study using lysozyme fibrils. Further, water in the sample appeared to be evaporated by the FEL irradiation. Although we could not investigate the vaporization dynamics in detail, it can be considered that thermal vapor bubble and pressure play some roles in the dissociation of the peptide aggregates based on the report [26]. From the infrared spectra and microscopic images obtained after the FEL irradiation, it can be considered that the peptide fibrils could be converted to the non-ordered structures by the FEL. Although the amide II and III irradiations were not tested for the short peptide, the above result indicates the significant conformational difference between lysozyme and the short peptide. Lysozyme is a functional enzyme and can form a globe fold in the active state. On the contrary, it can be considered that DFNKF peptide has no specific conformations in the solution before fibrillation. Given that the detailed conformational change of the peptide at atomic levels can be analyzed using NMR [27], structural analysis of the peptide fibrils will be further the subject of future investigation in our laboratory.

It can be considered that the infrared laser will be more safety than the organic solvents for the human body. Therefore, the above FEL oscillation system can be expected to be used for decreasing the toxicity of amyloid fibrils in pathological organs of amyloidosis patients, and in vivo study of the FEL will be next important subject.

Here, we would like to suggest two notable features of the above FEL irradiation system: One is that the target wavelength is, in principle, tunable within the mid-infrared region. For example, because the amide I bands of most pathological amyloid fibrils appear at lower wave numbers than those of non-toxic proteins, pathological proteins can be distinguished from normal proteins in terms of their infrared spectra [28]. Therefore, those pathological peptides or proteins can be selectively targeted by the above FEL oscillation system. The second point is that the FEL irradiation system can be connected to various microscopic instruments (as described in this study), and several target samples for dissociation can be observed in situ using those methods. For example, when aggregates of tumor cells from myeloma tissue is dissociated by the FEL, the structural changes after FEL irradiation can be analyzed in real time using an IR microscope connected with the FEL beam line. Such a bio-analytical experiment is now in the planning stage.

\section{Conclusion}

In conclusion, we have employed MIR-FEL to irradiate amyloid fibrils, and have demonstrated that the fibrils can be dissociated by the FEL irradiation tuned to the amide I band. Moreover, this result proves that the amyloid fibril structure is composed primarily of $\beta$-sheets formed between the amide backbones of the peptide. We expect this FEL irradiation system to be applied effectively in the dissociation of aggregates of various amyloid fibrils in biological samples. 


\section{Acknowledgements}

We thank Dr. Kanjiro Torigoe (Graduate School of Science and Technology, Tokyo University of Science) for providing technical support for the TEM analyses. We also thank Mr. Tetsuo Morotomi and Mr. Keiichi Hisazumi (Mitsubishi Electric System \& Service Co., Ltd.) for operating the FEL instrument, Mr. Wataru Kurashige for technical support for the MALDI-TOF MS analyses, and Mr. Jun Fujioka for his assistance in obtaining microscopic images. This work was supported in part by the Open Advanced Research Facilities Initiative of the Ministry of Education, Culture, Sport, Science, and Technology, Japan.

\section{References}

[1] Stroud, J.C., Liu, C., Teng, P.K. and Eisenberg, D. (2012) Toxic Fibrillar Oligomers of Amyloid- $\beta$ Have Cross- $\beta$ Structure. Proceedings of the National Academy of Sciences of the United States of America, 109, 7717-7722. http://dx.doi.org/10.1073/pnas.1203193109

[2] Zhu, H.-L., Meng, S.-R., Fan, J.-B., Chen, J. and Liang, Y. (2011) Fibrillization of Human Tau Is Accelerated by Exposure to Lead via Interaction with His-330 and His-362. ProS One, 6, Article ID: e25020. http://dx.doi.org/10.1371/journal.pone.0025020

[3] Smith, M.H., Miles, T.F., Sheehan, M., Alfieri, K.N., Kokona, B. and Fairman, R. (2010) Polyglutamine Fibrils Are Formed Using a Simple Designed $\beta$-Hairpin Model. Proteins, 78, 1971-1979. http://dx.doi.org/10.1002/prot.22713

[4] Panza, G., Stöhr, J., Dumpitak, C., Papathanassiou, D., Weiß, J., Riesner, D., Willbold, D. and Birkmann, E. (2008) Spontaneous and BSE-Prion-Seeded Amyloid Formation of Full Length Recombinant Bovine Prion Protein. Biochemical and Biophysical Research Communications, 373, 493-497. http://dx.doi.org/10.1016/j.bbrc.2008.06.059

[5] Ahmad, A., Uversky, V.N., Hong, D. and Fink, A.L. (2005) Early Events in the Fibrillation of Monomeric Insulin. The Journal of Biological Chemistry, 280, 42669-42675. http://dx.doi.org/10.1074/jbc.M504298200

[6] Spillantini, M.G., Schmidt, M.L., Lee, V. M.-Y., Trojanowski, J.Q., Jakes, R. and Goedert, M. (1997) $\alpha$-Synuclein in Lewy Bodies. Nature, 388, 839-840.

[7] Lu, M., Hiramatsu, H., Goto, Y. and Kitagawa, T. (2006) Structure of Interacting Segments in the Growing Amyloid Fibril of $\beta_{2}$-Microglobulin Probed with IR Spectroscopy. Journal of Molecular Biology, 362, 355-364. http://dx.doi.org/10.1016/j.jmb.2006.07.023

[8] Dobson, C.M. (2001) The Structural Basis of Protein Folding and Its Links with Human Disease. Philosophical Transactions of the Royal Society B, 356, 133-145. http://dx.doi.org/10.1098/rstb.2000.0758

[9] Gertz, M.A. (2013) Immunoglobulin Light Chain Amyloidosis: 2013 Update on Diagnosis, Prognosis, and Treatment. American Journal of Hematology, 88, 417-425. http://dx.doi.org/10.1002/ajh.23400

[10] Booth, D.R., Sunde, M., Bellotti, V., Robinson, C.V., Hutchinson, W.L., Fraser, P.E., Hawkins, P.N., Dobson, C.M., Radford, S.E., Blake, C.C.F. and Pepys, M.B. (1997) Instability, Unfolding and Aggregation of Human Lysozyme Variants Underlying Amyloid Fibrillogenesis. Nature, 385, 787-793.

[11] Edwards, G., Logan, R., Copeland, M., Reinisch, L., Davidson, J., Johnson, B., Maciunas, R., Mendenhall, M., Ossoff, R., Tribble, J., Werkhaven, J. and O’Day, D. (1994) Tissue Ablation by a Free-Electron Laser Tuned to the Amide II Band. Nature, 371, 416-419.

[12] Nomaru, K., Kawai, M., Yokoyama, M., Oda, F., Nakayama, A., Koike, H. and Kuroda, H. (2000) Optical Beam Transport System at FEL-SUT. Nuclear Instruments and Methods in Physics Research Section A, 445, 379-383.

[13] Oomens, J., Polfer, N., Moore, D.T., van der Meer, L., Marshall, A.G., Eyler, J.R., Meijer, G. and von Helden, G. (2005) Charge-State Resolved Mid-Infrared Spectroscopy of a Gas-Phase Protein. Physical Chemistry Chemical Physics, 7, 1345-1348. http://dx.doi.org/10.1039/B502322J

[14] Ovelmen-Levitt, J., Straub, K.D., Hauger, S., Szarmes, E., Madey, J., Pearlstein, R.D. and Nashold Jr., B.S. (2003) Brain Ablation in the Rat Cerebral Cortex Using a Tunable-Free Electron Laser. Lasers in Surgery and Medicine, 33, 81-92. http://dx.doi.org/10.1002/lsm.10197

[15] Annenkov, V.V., Kozlov, A.S., Danilovtseva, E.N., Basharina, T. N. and Petrov, A.K. (2013) Dissection of the Frustules of the Diatom Synedraacus under the Action of Picosecond Impulses of Submillimeter Laser Irradiation. European Biophysics Journal, 42, 587-590. http://dx.doi.org/10.1007/s00249-013-0913-1

[16] Austin, R.H., Xie, A., van der Meer, L., Redlich, B., Lindgård, P.-A., Frauenfelder, H. and Fu, D. (2005) Picosecond Thermometer in the Amide I Band of Myoglobin. Physical Review Letters, 94, 128101-1-4. http://dx.doi.org/10.1103/PhysRevLett.94.128101

[17] Hutson, M.S., Ivanov, B., Jayasinghe, A., Adunas, G., Xiao, Y., Guo, M. and Kozub, J. (2009) Interplay of Wavelength, Fluence and Spot-Size in Free-Electron Laser Ablation of Cornea. Optics Express, 17, 9840-9850. 
[18] Kawasaki, T., Fujioka, J., Imai, T. and Tsukiyama, K. (2012) Effect of Mid-Infrared Free-Electron Laser Irradiation on Refolding of Amyloid-Like Fibrils of Lysozyme into Native Form. The Protein Journal, 31, 710-716. http://dx.doi.org/10.1007/s10930-012-9452-3

[19] Zandomeneghi, G., Krebs, M.R.H., McCammon, M.G. and Fändrich, M. (2004) FTIR Reveals Structural Differences between Native $\beta$-Sheet Proteins and Amyloid Fibrils. Protein Science, 13, 3314-3321. http://dx.doi.org/10.1110/ps.041024904

[20] Reches, M., Porat, Y. and Gazit, E. (2002) Amyloid Fibril Formation by Pentapeptide and Tetrapeptide Fragments of Human Calcitonin. The Journal of Biological Chemistry, 277, 35475-35480. http://dx.doi.org/10.1074/jbc.M206039200

[21] Petruzziello, F., Zeppa, P., Catalano, L., Cozzolino, I., Gargiulo, G., Musto, P., D’Auria, F., Liso, V., Rizzi, R., Caruso, N., Califano, C., Piro, E., Musso, M., Bonanno, V., Falcone, A.P., Tafuto, S., Raimondo, F.D., Laurentiis, M.D., Pane, F., Palombini, L. and Rotoli, B. (2010) Amyloid in Bone Marrow Smears of Patients Affected by Multiple Myeloma. Annals of Hematology, 89, 469-474. http://dx.doi.org/10.1007/s00277-009-0857-9

[22] Bandekar, J. (1992) Amide Modes and Protein Conformation. Biochimica et Biophysica Acta-Protein Structure and Molecular Enzymology, 1120, 123-143.

[23] Thirumalai, D., Reddy, G. and Straub, J.E. (2012) Role of Water in Protein Aggregation and Amyloid Polymorphism. Accounts of Chemical Research, 45, 83-92. http://dx.doi.org/10.1021/ar2000869

[24] Zavalin, A., Hachey, D.L., Sundaramoorthy, M., Banerjee, S., Morgan, S., Feldman, L., Tolk, N. and Piston, D.W. (2008) Kinetics of a Collagen-Like Polypeptide Fragmentation after Mid-IR Free-Electron Laser Ablation. Biophysical Journal, 95, 1371-1381. http://dx.doi.org/10.1529/biophysj.107.122002

[25] Anfinsen, C.B. (1973) Principles That Govern the Folding of Protein Chains. Science, 181, 223-230.

[26] Wagner, W., Sokolow, A., Pearlstein, R. and Edwards, G. (2009) Thermal Vapor Bubble and Pressure Dynamics during Infrared Laser Ablation of Tissue. Applied Physics Letters, 94, 013901-1-3. http://dx.doi.org/10.1063/1.3063127

[27] Itoh-Watanabe, H., Kamihira-Ishijima, M., Javkhlantugs, N., Inoue, R., Itoh, Y., Endo, H., Tuzi, S., Saitô, H., Ueda, K. and Naito, A. (2013) Role of Aromatic Residues in Amyloid Fibril Formation of Human Calcitonin by Solid-State ${ }^{13} \mathrm{C}$ NMR and Molecular Dynamics Simulation. Physical Chemistry Chemical Physics, 15, 8890-8901. http://dx.doi.org/10.1039/c3cp44544e

[28] Botelho, H.M., Leal, S.S., Cardoso, I., Yanamandra, K., Morozova-Roche, L.A., Fritz, G. and Gomes, C.M. (2012) S100A6 Amyloid Fibril Formation Is Calcium-Modulated and Enhances Superoxide Dismutase-1 (SOD1) Aggregation. The Journal of Biological Chemistry, 287, 42233-42242. http://dx.doi.org/10.1074/jbc.M112.396416 